\title{
Use of gadobenate dimeglumine dynamic MRI for detection of early hepatocellular carcinoma in atypical hepatic focal lesions
}

\author{
Mohamed F. El-Gazzar ${ }^{1}$, Mohamed Ahmed Samy Kohla², Mahmoud M. El-Sakhawy ${ }^{3}$, Mohamed M. Husseiny ${ }^{3}$, Reda \\ R. H. Yousef ${ }^{4}$, Safinaz H. El-Shorbagy ${ }^{5}$
}

\begin{abstract}
${ }^{1}$ Department of Oncology, National Liver Institute, Menoufia University, Shebeen El-Kom 32511, Egypt. ${ }^{2}$ Department of Hepatology, National Liver Institute, Menoufia University, Shebeen El-Kom 32511, Egypt.

${ }^{3}$ Department of Radiology, National Liver Institute, Menoufia University, Shebeen El-Kom 32511, Egypt.

${ }^{4}$ Department of Radiodiagnosis, Faculty of Medicine, Al-Azhar University, Cairo 11651, Egypt.

${ }^{5}$ Department of Pathology, Faculty of Medicine, Tanta University, Tanta 31111, Egypt.
\end{abstract}

Correspondence to: Dr. Mohamed Ahmed Samy Kohla, Department of Hepatology, National Liver Institute, Menoufia University, Shebeen El-Kom 32511, Egypt.E-mail: dr_mohamedsamy@yahoo.com

How to cite this article: El-Gazzar MF, Kohla MAS, El-Sakhaw MM, Husseiny MM, Yousef RRH, El-Shorbagy SH. Use of gadobenate dimeglumine dynamic MRI for detection of early hepatocellular carcinoma in atypical hepatic focal lesions. Hepatoma Res 2017;3:123-8.

Article history:

Received: 16-03-2017

Accepted: 09-05-2017

Published: 26-06-2017

Key words:

Dynamic magnetic resonance

imaging,

multihance,

gadobenate dimeglumine,

early detection,

hepatocellular carcinoma

\begin{abstract}
Aim: Gadobenate dimeglumine (multihance) is a contrast medium which can be used not only as an extracellular contrast agent for dynamic imaging of the liver, but also as a liver specific agent for the acquisition of hepatobiliary-phase images which are more helpful in evaluation of small atypical hepatic focal lesions equal or less than $3 \mathrm{~cm}$. The authors tried to evaluate multihance dynamic magnetic resonance imaging (MRI) as a new modality in early detection of hepatocellular carcinoma (HCC). Methods: Thirty cirrhotic patients with small hepatic focal lesions (less than $3 \mathrm{~cm}$ in diameter), detected by imaging (ultrasound and triphasic computed tomography) were subjected to dynamic MRI with multihance contrast. All patients had a liver biopsy stained with heat shock protein 70 , glypican 3, and glutamine synthetase to confirm the diagnosis of HCC. Results: Eight out of 30 patients $(26.6 \%)$ with atypical focal lesions proved to have HCC by histology, whereas 7 out of 8 histologically proven HCC patients (87.5\%) were shown to have typical criteria on Multihance imaging. Conclusion: Multihance dynamic MRI is a promising diagnostic modality for detection of early HCC, however, future studies on large numbers of patients are warranted to precisely detect the sensitivity and specificity of this new modality.
\end{abstract}

\section{INTRODUCTION}

Hepatocellular carcinoma (HCC) is the most common primary tumor of the liver with a global incidence of approximately half a million cases per year. ${ }^{[1]}$ It is a rapidly fatal cancer that mostly affects persons in developing countries where hepatitis $B$ and hepatitis $C$ viruses are endemic. ${ }^{[2]}$ Cirrhosis is the most important risk factor for HCC; overall, one third of cirrhotic patients will develop HCC during their life time.$^{[3]}$ Portal supply gradually decreases in accordance with higher grades of malignancy of the nodules and finally disappears in 


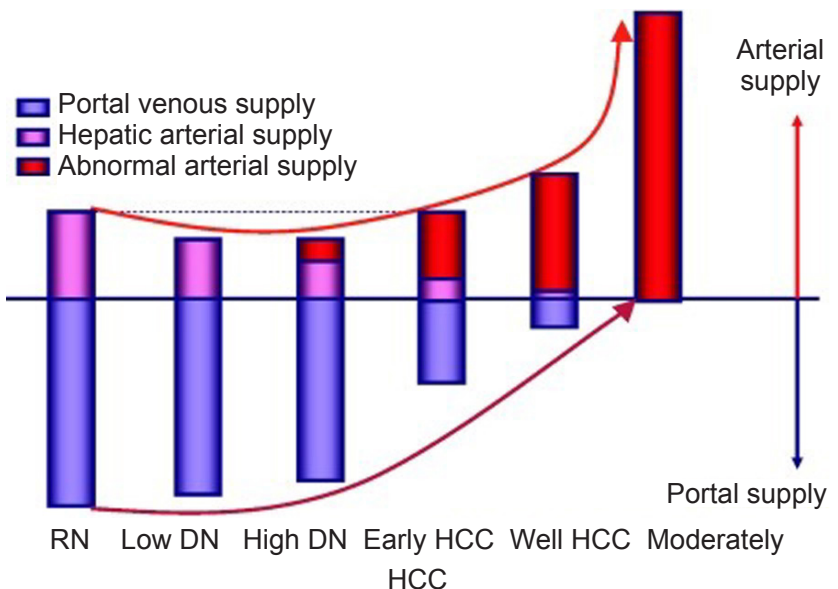

Figure 1: Multistep hepatocarcinogenesis. ${ }^{[4]} \mathrm{HCC}$ : hepatocellular carcinoma; DN:dysplastic nodule, RN: regenrating nodules

moderately differentiated HCCs [Figure 1]. ${ }^{[4]}$

Nowadays, early diagnosis of $\mathrm{HCC}$ is feasible only in $30-60 \%$ of cases in developed countries. However, the percentage is much less in developing countries. Early diagnosis enables the application of curative treatments. ${ }^{[5,6]}$ Thus there is an urgent need to identify better tools to characterize these atypical small lesions.

Diagnosis of small HCC lesions lacking typical hemodynamic criteria could be a challenge. Atypical enhancement patterns and hypovascular HCC lesions seen in a considerable number of HCC patients have led to around $35 \%$ false negative results in patients with tumors between $1-2 \mathrm{~cm}$ in diameter in triphasic computed tomography (CT) scan. ${ }^{[7-9]}$

Extracellular contrast agents in magnetic resonance imaging (MRI) are suited to liver imaging applications that require information obtained during the dynamic contrast-enhanced phase or excellent vascular visualization such as Gadobutrol (Gadovist US, Gadovist EU, Bayer) and Gadoversetamide (Optimark, Covidein) ${ }^{[10]}$

Hepatobiliary agents are suited to applications focusing on biliary visualization and distinguishing between hepatocytes and lesions not containing hepatocytes e.g. Gadobenate Dimeglumin (Multihance, Bracco), Gadoxetate Disodium (Eovist US). These agents have extracellular properties but also have affinity for hepatocytes. ${ }^{[11]}$

Multihance can be used not only as a non-specific extracellular contrast agent for dynamic imaging of the liver, but also as a liver specific agent for the acquisition of hepatobiliary-phase images. Lesions that contain functioning hepatocytes where hepatobiliary metabolism is mostly unaltered are expected to uptake multihance and excrete the compound into the bile. Such lesions are typically benign and usually appear isointense or hyperintense as compared to the normal liver parenchyma in the hepatobiliary phase of MRI. In contrast, lesions lacking functioning hepatocytes where hepatobiliary metabolism is blocked or inhibited are generally not able to uptake and excrete multihance into the bile. Such lesions are typically malignant and usually appear hypointense as compared to the normal liver parenchyma on the hepatobiliary phase of MRI. ${ }^{[10,11]}$ Therefore, multihance dynamic MRI is a potential promising diagnostic modality for detection of early HCC.

\section{METHODS}

This prospective study was conducted at National Liver Institute, Menoufia University. The study protocol was approved by the Institutional Review Board (IRB) and local ethical committee. A written informed consent was obtained from all participants in the study.

The study was conducted on 30 adult cirrhotic patients with atypical hepatic focal lesions. Patients were recruited from the outpatient HCC clinic of the National Liver Institute, Menoufia University. Patients were enrolled from October 2014 to June 2015.

\section{Inclusion criteria}

Cirrhotic patients with a single hepatic focal lesion not more than $3 \mathrm{~cm}$ in diameter detected by ultrasound with atypical enhancement pattern on triphasic CT scan and dynamic MRI and alpha fetoprotein (AFP) level less than $200 \mathrm{ng} / \mathrm{mL}$.

\section{Exclusion criteria}

Hepatic focal lesions more than $3 \mathrm{~cm}$, typical HCC lesions on triphasic CT, portal vein thrombosis, extrahepatic lymph node metastasis, metastatic lesions, AFP more than $200 \mathrm{ng} / \mathrm{mL}$ or previous HCC treatment. Patients with Child class $C$ decompensated cirrhosis in whom liver biopsy is contraindicated were excluded from the study.

Patient were selected on the basis of clinical presentation, liver function profile, complete blood picture, imaging procedures including ultrasonography, triphasic CT showing atypical enhancement pattern. Dynamic MRI with multihance contrast was used to detect HCC. Histopathological study of biopsy specimens from the lesions was performed using hematoxylin and eosin (HE) and immunohistochemical staining with glypican 3 (GLP3), heat shock protein 
Table 1: Patient and tumor characteristics

\begin{tabular}{lc}
\hline Variable & Number \\
\hline Mean diameter of nodules $(\mathrm{cm})$ & \\
HCC & 2.1 \\
Non-HCC & 1.2 \\
Laboratory tests & $35 \pm 28$ \\
ALT $(\mathrm{U} / \mathrm{L})$ & $45 \pm 30$ \\
AST $(\mathrm{U} / \mathrm{L})$ & $1.0 \pm 0.5$ \\
Total bilirubin $(\mathrm{mg} / \mathrm{dL})$ & $4.0 \pm 0.7$ \\
Albumin $(\mathrm{g} / \mathrm{dL})$ & $1.2 \pm 0.2$ \\
INR & $135 \pm 80$ \\
Platelets $\left(x 10^{9} / \mathrm{L}\right)$ & $6(75 \%)$ \\
HCC differentiation & $2(25 \%)$ \\
Well differentiated & $14.6 \pm 27.1$ \\
Moderately differentiated & \\
Poorly differentiation & \\
AFP (ng/mL) &
\end{tabular}

HCC: hepatocellular carcinoma; ALT: alanine transaminase; AST: aspartate transaminase; INR: international normalized ratio; AFP: alpha fetoprotein

70 (HSP70) and glutamine synthetase (GS). All the biopsy specimens were examined by the same pathologist.

\section{RESULTS}

A total of 30 patients with liver cirrhosis and atypical hepatic focal lesions were included in the study. The mean age was $56.1 \pm 11.6$ years (range $39-72$ years). The majority of patients were males (24 out of 30 patients, $80 \%$ ).

Chronic hepatitis $C$ virus infection was the cause of cirrhosis in 25 out of 30 patients $(83.3 \%)$, whereas chronic hepatitis $B$ was the cause in 5 patients $(16.7 \%)$. Nineteen patients had Child class A cirrhosis $(63.3 \%)$ while 11 patients had Child class B cirrhosis $(36.7 \%)$.

AFP was $8-121 \mathrm{ng} / \mathrm{mL}$ with mean $14.619 \pm 27.187$ [Table 1]. The mean diameter of the nodules was $19 \mathrm{~mm}$. Pathological examination of liver biopsies by HE and immunostaining GLP3, GS and HSP70
Table 2: MRI with multihance findings in all patients

\begin{tabular}{lcc}
\hline Finding description & HCC patients & Non-HCC patients \\
\hline Hypointenisity on T1 & 4 & 5 \\
Hyperintensity on T2 & 5 & 6 \\
Arterial hyperintensity & 7 & 7 \\
Delayed hypointensity & 0 & 2 \\
Hepatobiliaryhypointensity & 7 & 5 \\
Rim enhancement & 2 & 1 \\
\hline
\end{tabular}

HCC: hepatocellular carcinoma; MRI: magnetic resonance imaging

showed that only 8 patients $(26.6 \%)$ had at least 2 immunostaining markers positive for HCC [Figure 2]. Out of 8 patients with histologically proven HCC, 6 $(75 \%)$ had a well differentiated HCC and 2 (25\%) had a moderately differentiated HCC [Table 1].

Out of 30 patients, 6 patients (20\%) had regenerating nodules, 9 patients $(30 \%)$ had high grade dysplastic nodules and 7 patients $(23 \%)$ had low grade dysplastic nodules.

In triphasic CT, arterial enhancement was seen in 6 $\mathrm{HCC}$ and 5 non-HCC patients, portal venous washout was not seen in any HCC patient but seen in 2 nonHCC patients, delayed hypointensity was not seen in any HCC patient but seen in 1 non-HCC patient.

MRI using multihance [Figure 3] showed that 7 patients $(23.3 \%$ of all atypical hepatic focal lesions; $87.5 \%$ of HCC patients) had a hypevascular hepatobiliary phase (HBP) hypointense HCC, 7 patients had non-hyper vascular HBP hypointense focal lesions. Using multihance MRI, 4 HCC patients and 5 non-HCC patients showed hypointensity in T1, 5 HCC patients and 6 non-HCC patients showed hyper intensity in T2 [Table 2]. Arterial enhancement was seen in $7 \mathrm{HCC}$ and 7 non-HCC patients, delayed hypointensity was not seen in any $\mathrm{HCC}$ patients but seen in 2 non-HCC patients, HBP hypointensity was seen in $7 \mathrm{HCC}$ and 5 non-HCC patients. Rim enhancement was seen in delayed phase in $2 \mathrm{HCC}$
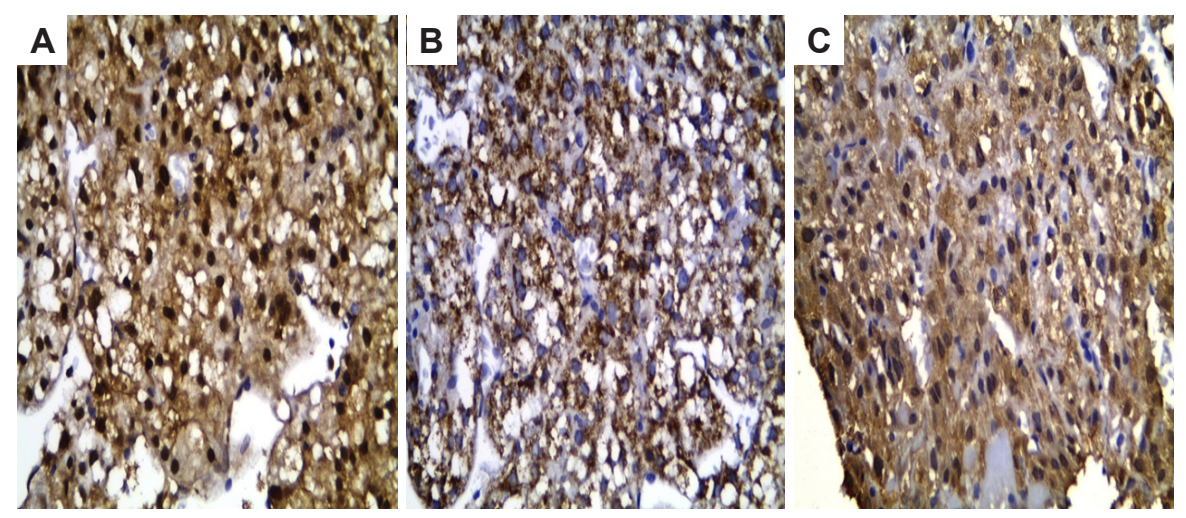

Figure 2: Immunohistochemical staining (x400) in well differentiated HCC showing: (A) high nucleocytoplasmic heat shock protein 70 expression; (B) high cytoplasmic glypican 3 expression; (C) high cytoplasmic glutamin synthetase expression. HCC: hepatocellular carcinoma 
Table 3: Combinations of different phases of MRI

\begin{tabular}{|c|c|c|}
\hline Phase & HCC patients & Non-HCC patients \\
\hline $\begin{array}{l}\text { Arterial phase hyperintensity } \\
\text { HBP hypointense }\end{array}$ & 7 & 4 \\
\hline $\begin{array}{l}\text { Hypovascular, HBP } \\
\text { hypointense }\end{array}$ & 1 & 7 \\
\hline $\begin{array}{l}\text { Arterial phase hyperintensity } \\
\text { HBP hypointense, rim } \\
\text { enhancement }\end{array}$ & 2 & 1 \\
\hline
\end{tabular}

HCC: hepatocellular carcinoma; HBP: hepatobiliary phase; MRI: magnetic resonance imaging

patients and 1 non-HCC patient [Table 2].

On combining different phases, it was found that 5 HCC patients showed T2 hyper-intensity and hypervscular HBP hypointense lesions, those findings were not detected in non-HCC patients. Seven nonHCC patients showed hypovascular HBP hypointense lesions versus $1 \mathrm{HCC}$ patient [Table 3]. Two HCC patients showed hypervascular HBP hypointense and rim enhancement at delayed phase versus 1 non-HCC patient [Table 3]. The sensitivity on combining the arterial phase hyperintensity and HBP hypointensity was $87.5 \%$ and specificity was $82.8 \%$.

\section{DISCUSSION}

Gadobenate dimeglumine is not a novel agent but the group of atypical HCC selected is a challenging group for diagnosis. Guidelines for diagnosis of atypical cases of HCC are not yet well established and need further studies using different imaging modalities for early diagnosis thereby optimizing treatment outcome.

In our study, 8 out of 30 cirrhotic patients with atypical hepatic focal lesions on triphasic CT, were diagnosed as $\mathrm{HCC}$ based on positive immunostaining of at least 2 HCC biomarkers (GLP3, HSP70, or GS) according to the international consensus group for hepatocellular neoplasia. Seven out of these 8 lesions were diagnosed by gadobenate dimeglumine-enhanced MRI (multihance) as HCC, showing hyperintensity in the arterial phase and hypointensity in the HBP (according to the latest guidelines for diagnosis of $\mathrm{HCC}$ including those of the Japan Society of Hepatology (JSH), ${ }^{[12]}$ the Korean Liver Cancer Study Group (KLCSG), the National Cancer Center (NCC),${ }^{[13]}$ and the Liver Imaging Reporting and Data System (LI-RADS). ${ }^{[14]}$

According to the updated $2014 \mathrm{JSH}$ guidelines, noninvasive diagnosis of $\mathrm{HCC}$ can be made using a hepatobiliary contrast if a lesion shows: (1) arterial hypervascularity and venous washout or (2) arterial hypervascularity without venous washout, but with hypointensity on the HBP. ${ }^{[15]}$
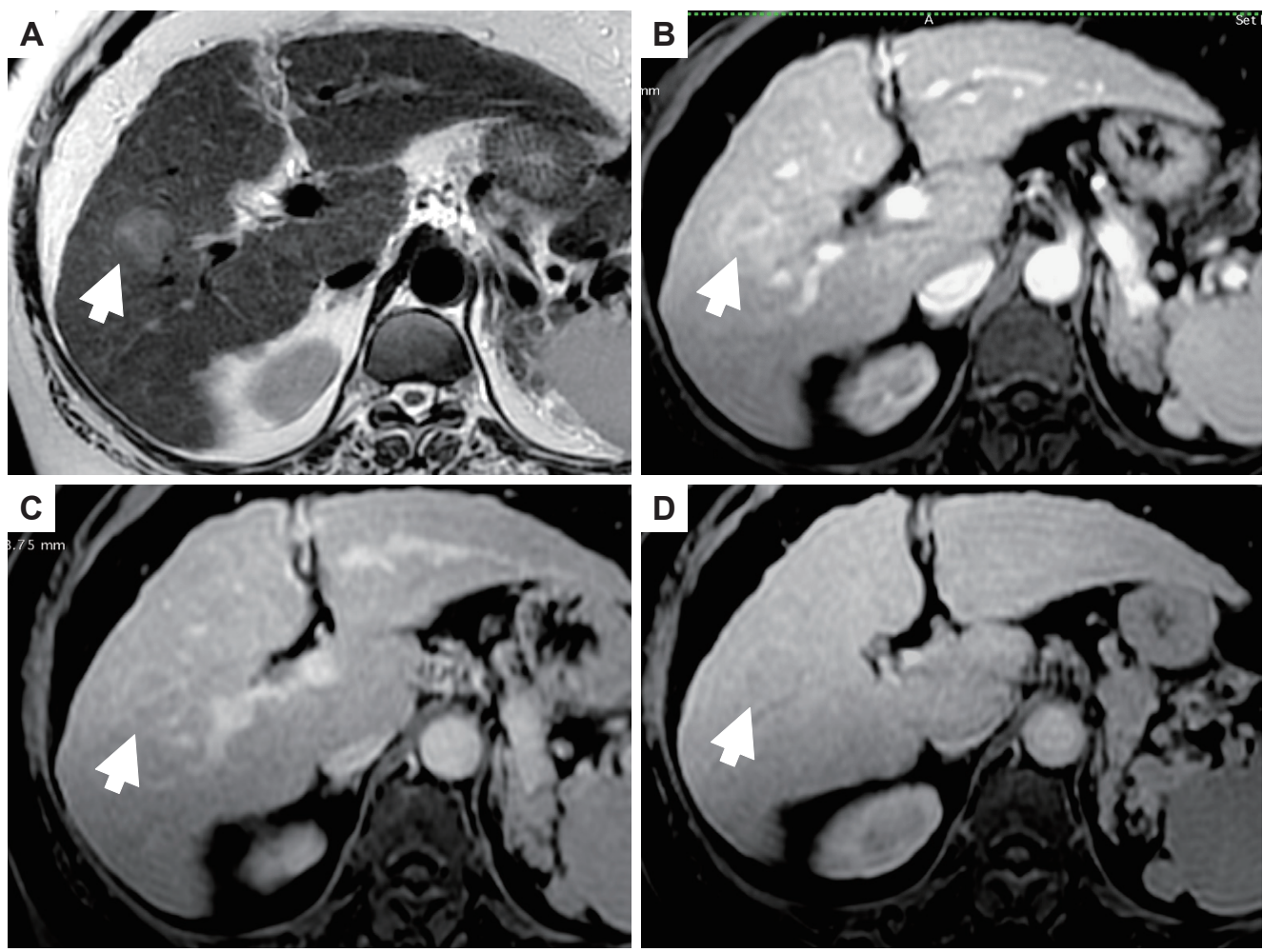

Figure 3: Trans-axial magnetic resonance images of the liver demonstrates right lobe focal lesion (arrow). It appears hyper-intense on T2 image (A). The arterial phase (B) shows faint enhancement in the lesion with washout on the arterio-portal phase (C) and the appearance of capsule. The lesion is hypo-intense to the liver parenchyma on the hepato-biliary phase (D). This lesion proved to hepatocellular carcinoma on histopathology 
Most guidelines on the management of $\mathrm{HCC}$ recommend four-phase multi-detector $\mathrm{CT}$ and/ or contrast-enhanced MRI as standard imaging modalities for diagnosis of HCC, based on the typical dynamic pattern of arterial enhancement and washout on the portal/delayed phases. ${ }^{[16]}$ Some HCC lesions, especially those less than $2-3 \mathrm{~cm}$ in diameter, lack typical hemodynamic criteria, making diagnosis of $\mathrm{HCC}$ a big challenge. Atypical enhancement patterns were seen in around $30 \%$ of HCC patients in many studies and hypovascular HCC lesions have led to around $35 \%$ of false negative results in tumors $1-2 \mathrm{~cm}$ in diameter. ${ }^{[0]}$

$\mathrm{HCC}$ is a highly vascular tumor characterized by neoangiogenesis, which contributes to the high rate of metastasis and dismal prognosis. On microscopic examination, HCC displays marked vascular abnormalities, and aberrant microvasculature in the form of arteriogenesis and capillarization. Arteriogenesis is defined as the growth of functional collateral arteries covered with smooth muscle cells from pre-existing arteries. HCC is mainly supplied by hepatic arteries, while in normal liver parenchyma, regenerative and dysplastic nodules are mainly supplied by the portal vein. ${ }^{[4,17]}$

MR imaging with hepatobiliary contrasts such as multihance (gadobenate dimeglumine) and primovist (gadolinium ethoxybenzyl diethylenetriamine pentaacetic acid, Gd-EOB-DTPA, or gadoxetic acid) was found to be highly sensitive in HCC diagnosis. ${ }^{[18]}$ In one study, all HCC lesions were identified with gadoxetic acid, whereas three of 56 HCCs were not identified on dual-contrast MRI. In 59 patients imaged with gadoxetic acid, $10.7 \%$ of detected HCCs were detected only on hepatocellular phase images. ${ }^{[19]}$

In one study evaluating 86 nodules, the diagnostic ability of gadoxetic acid was significantly higher than that of multi-detector triphasic CT for tumors less than $2 \mathrm{~cm}$ in diameter. There was no significant difference in the detection of hypervascular HCCs between hepatobiliary phase images of gadoxetic acid-enhanced MRI (43/45: 96\%) and dynamic CT (40/45: $89 \%$ ), whereas the detection sensitivity of hypovascular tumors by gadoxetic acid-enhanced MRI was significantly higher than that by dynamic CT (39/41: 95\% vs. 25/41: $61 \%, P=0.001)$. Gadoxetic acid enhancement ratios were decreased in parallel with the degree of differentiation in dysplastic nodules and HCCs. ${ }^{[20]}$

In another study, imaging findings of a prospective and consecutive sample of $1-2 \mathrm{~cm}$ nodules detected at surveillance ultrasound suggest that the newly proposed criteria for nodules fitting the American Association for the Study of Liver Diseases practice guidelines or having 3 or more findings in gadobenate dimeglumine MRI can be a useful alternative providing a significant improvement in sensitivity while maintaining high specificity for diagnosis of HCC. ${ }^{[21]}$ In two other studies using gadobenate dimeglumine-enhanced MR, small nodules that show enhancement on arterial phase and occult on the portal and equilibrium phase images as well as on the T1 and T2-weighted images are more likely to be HCC in patients with hepatitis B-induced cirrhosis ${ }^{[22,23]}$ which are in accordance with our results.

Triphasic CT and gadolinium MRI are not ideal tools for diagnosis of atypical HCC as they depend only on extracellular contrast. However, gadobenate dimeglumine is a dual extra- and intracellular contrast which can assess not only vascular changes in atypical nodules but also enzymatic activity within hepatocytes that can develop earlier than vascular changes.

The cost of gadobenate dimeglumine contrast is about 30 euros per case, while that of gadolinium (routinely used in dynamic MRI) is around 15 euros per case, and the average cost of triphasic CT contrast, ultravist, is about 10 euros. Unlike triaphasic CT and gadolinium-enhanced MRI which are unreliable tools for diagnosis of small atypical HCC (almost one third of HCC cases), gadobenate dimeglumine-enhanced MRI was found to have higher sensitivity (87.5\%) and specificity (82.8\%) with an accurate diagnosis of more than $80 \%$ of atypical HCC in our study. Atypical $\mathrm{HCC}$ are usually well differentiated lesions with a better response to treatment, therefore, gadobenate dimeglumine MRI is likely to be more cost effective compared to other imaging modalities which warrants further studies.

In conclusion, gadobenate dimeglumen is a promising hepatobiliary contrast, which can potentially improve the non-invasive diagnosis of early atypical small HCC. The encouraging results from our pilot study warrants further confirmation by larger multicenter studies, as well as cost effective analysis of multihance MRI.

\section{Authors' contributions}

Study design: M.F. El-Gazzar, M.A.S. Kohla

Data analysis and manuscript preparation: M.F. ElGazzar, M.A.S. Kohla, M.M. El-Sakhawy, M.M. Husseiny, R.R.H. Yousef, S.H. El-Shorbagy

Critical review of manuscript: M.F. El-Gazzar, M.A.S. Kohla, M.M. El-Sakhawy 


\section{Financial support and sponsorship}

This study was supported by the National Liver Institute, Menoufia University, Egypt.

\section{Conflicts of interest}

There are no conflicts of interest.

\section{Patient consent}

A written informed consent was obtained from all participants in the study.

\section{Ethics approval}

The study protocol was approved by the Institutional Review Board (IRB) and local ethical committee of the National Liver Institute, Menoufia University.

\section{REFERENCES}

1. Bruix J, Sherman M. Management of hepatocellular carcinoma: an update. Hepatology 2011:53:1020-2.

2. Shen YC, Hsu C, Cheng CC, Hu FC, Cheng AL. A critical evaluation of the preventive effect of antiviral therapy on the development of HCC in patients with chronic hepatitis C or B. Oncology 2012;82:275-89.

3. Stangovanni A, Prati GM, Fasani P, Ronchi G, Romeo R, Manini M, Del Ninno E, Morabito A, Colombo M. The natural history of compensated cirrhosis due to hepatitis $\mathrm{C}$ virus: a 17-year cohort study of 214 patients. Hepatology 2006; 34:1303-10.

4. Matsui O, Kobayashi S, Sanada J, Kouda W, Ryu Y, Kozaka K, Kitao A, Nakamura K, Gabata T. Hepatocellular nodules in liver cirrhosis: hemodynamic evaluation (angiography-assisted CT) with special reference to multi-step hepatocarcinogenesis. Abdom Imaging 2011;3:264-72.

5. Kudo M. Early hepatocellular carcinoma: definition and diagnosis. Liver Cancer 2013;2:69-72.

6. Llovet JM, Bruix J. Novel advancement in management of hepatocellular carcinoma in 2008. J Hepatol 2008;48:S20-37.

7. Ricke J, Seidensticker M, Mohnike K. Noninvasive diagnosis of $\mathrm{HCC}$ in cirrhotic liver: current guidelines and future prospects for radiological imaging. Liver Cancer 2012;1:51-8.

8. Bolondi L, GaianiS, Celli N, Glofieri R, Grigioni WF, Leoni S, Venturi AM, Piscagila F. Characterization of small nodules in cirrhosis by assessment of vascularity: the problem of hypovascular hepatocellular carcinoma. Hepatology 2005;42:27-34.

9. Kim I, Kim MJ. Histological characteristics of HCC showing atypical en-hancement pattern on 4-phase MDCT examination. Korean $J$ Radiol 2012;13:586-93.

10. Bartolozzi C, Crocetti L, Lenicioni R, Cioni D, Della Pina C, Campani D. Biliary and reticuloendothelial impairment in hepatocarcinogenesis: the diagnostic role of tissue-specific MR contrast media. Eur Radiol 2007;17:2519-30.

11. Kim JI, Lee JM, Choi JY, Kim YK, Kim SH, Lee JY, Han JK, Choi
BI . The value of gadobenate dimeglumine-enhanced delayed phase MRI for characterization of hepatocellular nodules in the cirrhotic liver. Invest Radiol 2008;43:202-10.

12. Kudo M, Matsui O, Izumi N, Iijima H, Kadoya M, Imai Y. Liver cancer study group of Japan surveillance and diagnostic algorithm for hepatocellular carcinoma proposed by the liver cancer study group of Japan: 2014 update. Oncology 2014;87 Suppl 1:7-21.

13. Lee JM, Park JW, Choi BI. 2014 KLCSG-NCC Korea practice guidelines for the management of hepatocellular carcinoma: HCC diagnostic algorithm. Dig Dis 2014;32:764-77.

14. Mitchell DG, Bruix J, Sherman M, Sirlin CB. LI-RADS (liver imaging reporting and data system): summary, discussion, and consensus of the LI-RADS management working group and future directions. Hepatology 2015;61:1056-65.

15. Kudo M, Matsui O, Izumi N, Iijima H, Kadoya M, Imai Y, Okusaka T, Miyayama S, Tsuchiya K, Ueshima K, Hiraoka A, Ikeda M, Ogasawara S, Yamashita T, Minami T, Yamakado K; Liver Cancer Study Group of Japan. JSH Consensus-Based Clinical Practice Guidelines for the Management of Hepatocellular Carcinoma: 2014 Update by the Liver Cancer Study Group of Japan. Liver Cancer 2014;3:458-68.

16. Bota S, Piscaglia F, Marinelli S, Pecorelli A, Terzi E, Bolondi L. Comparison of international guidelines for noninvasive diagnosis of hepatocellular carcinoma. Liver Cancer 2012;1:190-200.

17. Sanz-Cameno P, Trapero-Marugán M, Chaparro M, Jones EA Moreno-Otero R. Angiogenesis: from chronic liver inflammation to hepatocellular carcinoma. J Oncol 2010;2010:272170.

18. Kim YK, Kim CS, Han YM, Park G. Detection of small hepatocellular carcinoma: can gadoxetic acid-enhanced magnetic resonance imaging replace combining gadopentetatedimeglumineenhanced and superparamagnetic iron oxide-enhanced magnetic resonance imaging? Invest Radiol 2010;45:740-6.

19. Ahn SS, Kim MJ, Lim JS, Hong HS, Chung YE, Choi JY. Added value of gadoxetic acid-enhanced hepatobiliary phase MR imaging in the diagnosis of hepatocellular carcinoma. Radiology 2010;255:459-66.

20. Inoue T, Kudo M, Komuta M, Hayaishi S, Ueda T, Takita M, Kita S, Hatanaka K, Yada N, Hagiwara S, Chung H, Sakurai T, Ueshima K, Sakamoto M, Maenishi O, Hyodo T, Okada M, Kumano S, Murakami T. Assessment of Gd-EOB-DTPA-enhanced MRI for $\mathrm{HCC}$ and dysplastic nodules and comparison of detection sensitivity versus MDCT. J Gastroenterol 2012;47:1036-47.

21. Kim TK, Lee KH, Jang HJ, Jacks LM, Menesez RJ, Park SH, Yazd L, Sherman M, Khalil K. Analysis of gadobenatedimenglumineenhanced MR findings for characterizing small (1-2) hepatic nodules inpatients at high risk for hepatocellular carcinoma. Radiology 2011;259:730-8

22. Kim YK, Lee YH, Kwak HS, Kim CS, Han YM. Clinical implication of small $(<20 \mathrm{~mm})$ enhancing hepatic nodules observed only during three-dimensional gadobenatedimenglumine-enhanced arterial-phase MRI of the hepatitis B virus-induced mild cirrhosis. Clin Imaging 2008;32:453-9.

23. Quaia E, Pizzolato R, De Paoli L, Angileri R, Ukmar M, Cova MA. Arterial enhancing-only nodules less than $2 \mathrm{~cm}$ in diameter in patients with liver cirrhosis: predictors of hepatocellular carcinoma diagnosis on gadobenatedimenglumine-enhanced MR imaging. $J$ Mag Reson Imaging 2013;37:892-902. 